\title{
Solar initiative at Oukaimeden Observatory
}

\author{
Zouhair Benkaldoun ${ }^{1}$, Jonathan J. Makela ${ }^{2}$ and John W. Meriwether ${ }^{3}$ \\ ${ }^{1}$ Oukaimeden Observatory, LPHEA, Cadi Ayyad University, Morocco \\ email: zouhair@uca.ma \\ ${ }^{2}$ Department of Electrical and Computer Engineering, University of Illinois at \\ Urbana-Champaign, Urbana, Illinois, USA \\ email: jmakela@illinois.edu \\ ${ }^{3}$ Department of Physics and Astronomy, Clemson University, Clemson, South Carolina, USA \\ email: meriwej@clemson.edu
}

\begin{abstract}
The solar research program at Oukaimeden Observatory started in 1988 with the helioseimological IRIS network. The Moroccan researchers involved in this research have analyzed solar observations in order to detect and characterize the solar sphere modes of oscillations. In the coming year, the researchers at the Oukaimeden Observatory will add new research capabilities by joining the International Space Weather Initiative (ISWI), installing a suite of optical instruments, comprising a Remote Equatorial Nighttime Observatory of Ionospheric Regions (RENOIR). The scope and objectives to be achieved in this proposed project are to:

- deploy a Fabry-Perot interferometer and wide-angle imaging system to the Observatoire Astronomique Universitaire de LOukaimeden;

- train students and researchers from Cadi Ayyad University on the operation of the equipment and related analysis techniques;

- collect and analyze data from the equipment to study properties of upper-atmospheric winds and temperatures and how they relate to the occurrence of space weather; and

- develop an international collaboration network with other researchers using similar instrumentation in Brazil and Peru. We will present here the plan we intend to develop for the Moroccan solar program in connection with ISWI.
\end{abstract}

Keywords. instrumentation: interferometers, plasmas.

\section{Introduction}

Space weather is a relatively new field of study and encompasses understanding how the near-space environment responds to forcing from lower-atmosphere weather systems as well as conditions on the sun. Although the specific effects of space weather including power grid failures, communication outages, and navigation errors when using spacebased navigation systems such as GPS are local in nature, understanding and predicting their occurrence requires a global view of the environment. Recognizing this, the United Nations has sponsored the International Space Weather Initiative (ISWI), a multinational program focused on advancing the understanding of space weather through the global deployment of space weather sensors.

To address the need for global coverage of measurements, the ISWI has brought together a variety of instrument providers and hosts from around the world to build an unprecedented observing network for space weather science. In comparison to other regions of the globe, the African sector has received the least amount of attention in terms of ground-based ionospheric measurements. Most studies that present statistics for the African sector are based upon satellite data. However, ground-based data are crucial for providing data that are not biased by a particular satellite's orbital characteristics. 
The Remote Equatorial Nighttime Observatory of Ionospheric Regions (RENOIR) experiment, comprised of ground-based optical instruments designed to study the thermosphere and ionosphere system, will add a new measurement capability needed to understand space weather over Africa. RENOIR will be deployed to the Observatoire Astronomique Universitaire de LOukaimden near Marrakech, Morocco. This site was chosen due to its already existing scientific infrastructure, as well its exceptional optical seeing conditions (Benkaldoun et al., 2005). Results from RENOIR in Morocco will complement similar installations in Brazil and Peru for understanding the global distribution of space weather events.

\section{RENOIR Instrumentation}

The instruments included in a RENOIR station allow for the study of the background thermospheric conditions (winds and temperatures) as well as the space weather effects in the ionosphere (e.g., equatorial plasma bubbles, medium-scale traveling ionospheric disturbances). Two instruments will be deployed to Oukaimeden Observatory: a FabryPerot interferometer (FPI) and a wide-angle imaging system. Both instruments make observations of naturally occurring airglow emissions caused by the dissociative recombination of $\mathrm{O}_{2}^{+}$, which can be used as a tracer of dynamics in the thermosphere/ionosphere system at $\sim 250 \mathrm{~km}$ altitude in the Earth's atmosphere. This emission occurs at a wavelength of $630.0 \mathrm{~nm}$.

The FPI will provide high-resolution spectral measurements of the $630.0-\mathrm{nm}$ emission from which the thermospheric winds and temperatures can be estimated. Data will be analyzed using routines developed at the University of Illinois (Makela et al., 2011). The wide-angle imaging system provides measurements of ionospheric structures occurring in an approximately $1000 \times 1000 \mathrm{~km}^{2}$ region above the observing site. Similar systems previously deployed in other parts of the world have demonstrated their utility for elucidating the physics of space weather processes (e.g., Makela and Miller, 2011, Makela et al., 2010, Meriwether et al., 2011). Data products will include estimates and statistical uncertainties of the thermospheric neutral winds and temperatures as well as individual images of thermospheric/ionospheric structure. Approximately 100 GB of data per year is expected to be generated.

\section{Conclusions}

The anticipated long-term deployment of RENOIR to Morocco represents the first opportunity to study the climatology of winds and temperatures from the African continent over multiple seasons and years. The scientific output of RENOIR in Morocco will be augmented by the other ISWI instrumentation that has been recently deployed to Africa.

Furthermore, this program will train doctoral degree candidates in the solar physics field in general, and the Sun-Earth environment in particular. Instrumentation and collaborations introduced by this cooperation will allow us to contribute to the development of the Oukaimden Observtory as a scientific and training institute unique in the region.

\section{References}

Benkaldoun, Z., Abahamid, A., El Azhari, Y., \& Lazrek, M. 2005, A\& A, 441, 839

Makela, J. J., Meriwether, J. W., Huang, Y., \& Sherwood, P. J. 2011, Applied Optics, 50, 4403

Makela, J. J. \& Miller, E. S. 2010, in Abdu, M. A. and D. Pancheva, editors, Aeronomy of the Earth's Atmosphere and Ionosphere, 239

Meriwether J. W., Makela, J. J., Huang, Y., Fisher, D. J., Buriti, R. A., Medeiros, A. F., \& Takahashi, H. 2011, J. Geophys. Res., 116, A04322 